\title{
The reflectrometry as a multi-function diagnostic to characterize turbulence of fusion plasmas
}

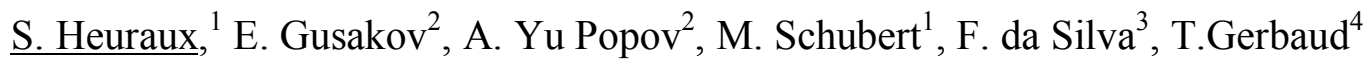 \\ ${ }^{1}$ LPMIA, UHP Nancy_Université BP239 54506 Vandoeuvre cedex-France \\ ${ }^{2}$ Ioffe Institute Politekhnicheskaya 26, 194021 St.Petersburg, Russia \\ ${ }^{3}$ Centro de Fusão Nuclear EURATOM / IST Av. Rovisco Pais, 1049-001 Lisboa, Portugal \\ ${ }^{4}$ Association Euratom-CEA_Cadarache 13108 St Paul-lez-Durance-France
}

\section{stephane.heuraux@lpmi.uhp-nancy.fr}

The understanding of the role played by the turbulent transport is a key point for industrial fusion energy production, for that information is needed on the density fluctuations, amplitude, frequency and wavenumber spectra, correlation lengths and so on. The reflectometry is a versatile tool able to provide such turbulence characteristics ${ }^{1}$. The absolute amplitude of the density fluctuations associated to the turbulence can be obtained with different kinds of reflectometers. However some theoretical works have to be done to interpret correctly the reflectometer data and to evaluate the capabilities of reflectometry systems. In the framework of the Lorraine-Russia ARCUS program, some of them have been realized $^{2-3}$, others are underway ${ }^{4-5}$ and new ones are under discussion. Evaluations of different kinds of reflectometer will be presented and a short part will be devoted to a new data processing method applied on the radial correlation reflectometry data able to extract the radial wavenumber spectrum of the turbulence ${ }^{6-8}$. To conclude, a brief review of other possibilities for reflectometers will be presented especially in the domain of the turbulence dynamics.

The fluctuation reflectometer working at fixed frequency has been the first use of a reflectometer as diagnostic providing information on density fluctuation $\delta$. Later technical progresses have permitted to sweep the frequency fast enough to reach steady-state density fluctuations and to give measurements able to be used to reconstruct accurate density profiles $^{9}$. Now the density profile can be obtained with a spatial resolution better than $1 \mathrm{~cm}$ each $25 \mu \mathrm{s}^{1}$, which gives the possibility to follow fast events, such as, edge localized modes (ELM) or a massive gas injection or Magneto Hydro Dynamics (MHD) activities. It is also possible to extract characteristics (radial wavenumber spectrum, density fluctuation profile) of the turbulence using a fast frequency sweep reflectometer assuming that the density fluctuations are frozen during the sweeping time ${ }^{6,8}$. However more work has to be done in this field to determine properly the validity domain of this method for high amplitude of density fluctuations. At this moment for X-mode reflectometer, this method is still working up to $\delta \mathrm{n} / \mathrm{n}=15 \%$ in the case of Tore Supra reflectometers for the measurements of the absolute density fluctuation profile. For O-mode the reconstructed radial wavenumber spectrum, obtained by using analytic expression in the Born approximation, is distorted by non-linear effects when the amplitude of density fluctuation reaches the limit of the Born approximation $^{6}$. This limit can be found in the Afeyan's paper ${ }^{10}$. Some studies have to be done for studying the cases where magnetic islands and turbulence are present at the same time. The magnetic islands modulate in time the local value of the density gradient length and at this moment only a time averaged value of the density gradient is used to evaluate the absolute density fluctuation profile. That gives hills in density fluctuation profiles in the vicinity of rational surfaces ${ }^{8}$. Some questions remain open for the different kinds of reflectometers. For the fluctuation reflectometer working at fixed frequency, is the absolute density fluctuation measurements always available and is it a function of the wavenumber 
spectrum properties? In the case of radial correlation reflectometry working with two different frequencies with a variable frequency different, is it still possible to use it when the non-linear effects are dominant? For Doppler reflectometry, what is the role of the forward scattering on the measurement, what can be the signature of the forward scattering? There is a more general question on the origin of the phase jumps. In the following, partial answers will be given to these questions corresponding to the state of art. Other open questions exist in the reflectometry field but are not connected to the ARCUS program.

The fluctuation reflectometer is routinely used to measure the absolute density fluctuation amplitude in the vicinity of the cut-off layer and the data processing assumes that the level of turbulence can be extracted from the RMS value of the phase fluctuations using an expression obtained in the Born approximation with an ad hoc or effective wavenumber ${ }^{1}$ or a more sophisticate expression including the non-linear effects and the radial correlation length of the turbulence ${ }^{14}$. It has been verified that the density fluctuation profile plays also a role to determine the absolute value of the density fluctuation amplitude ${ }^{4}$. In the case of Tore Supra tokamak, the fluctuation reflectometer is unable to provide the absolute value of the density fluctuation in the vicinity of the cut-off layer due to the Bragg backscattering contributions coming from the edge of the plasma where the amplitude of density fluctuation is of the order of $15 \%$. This forbidden zone is described in details in the reference 4 . To evaluate properly the effect of long wavelengths the simulation requirements are important the wavenumber resolution should be small enough $\Delta \mathrm{k} \mathrm{L}<10^{-1}$ to have accurate results better than $1 \%$ where $\mathrm{L}$ is the density gradient length, which corresponds to long computation time and a lot of memory at the limit of a workstation (4GBytes) for 1D simulation. These simulations have permitted to validate the improved formula ${ }^{14}$ in various situations in the case a Gaussian wavenumber spectrum. More realistic cases with non-Gaussian spectra are underway and will be submitted to publication soon.

The radial correlation reflectometer shows an interesting behavior. At low amplitude of density fluctuation the correlation function follows a logarithmic evolution and at high amplitude gives radial correlation length much smaller than the input correlation lengths of the density fluctuation ${ }^{3}$ when multiple small angle scattering takes place. At high turbulence level and/or in large reactor scale plasmas using radial correlation reflectometry, the coherence decay for growing cut offs separation is dependent on the density fluctuation level and this property can be used for turbulence characterization. A specific behavior of scattering efficiency at long scales inversely proportional to radial wave number is universal persisting both in $1 \mathrm{D}$ and $2 \mathrm{D}$ linear radial correlation reflectometry theory ${ }^{4}$ and for arbitrary density and turbulence profiles. A method of turbulence radial wave number spectrum reconstruction accounting for the universal scattering efficiency dependence on fluctuation wave number has been proposed and tested with 1D full-wave code. However this procedure needs to be evaluated in the case where non-linear effects take place and the reflected signals are noisy due to fast electron radiation for instance.

Doppler reflectometry becomes a very useful tool to characterize the turbulence especially to evaluate the poloidal velocity of the density fluctuation and to access the poloidal wavenumber spectrum. To extract the poloidal wavenumber spectrum some assumptions are done such as the backscattered signal comes from the vicinity of the turning point with constant efficiency for O-mode. This assumption falls down for the X-mode due to a decay of efficiency with an increase value of the fluctuation wavenumber as shown by simulation. In this description the forward scattering effect is missing. So to interpret correctly the results obtained by Doppler reflectometry a model has been built and determine if the experimental situation is subject to non-linear contribution associated to the forward 
scattering $^{15}$. A comparison between theory and simulation of a Gaussian wavenumber input spectrum shows that the behavior is well described in terms of amplitude of backscattered wave and frequency shift versus the amplitude of the density fluctuation but the scattered power values remain different by a factor of two or more. To make a full the comparison the time series should be very long of the order of one million of time steps. However these studies have permitted to find typical signatures if forward scattering dominates, the backscattered signal becomes bursty and the frequency shift is a function of the turbulence level, in opposite when the Bragg backscattering dominates the frequency shift stays constant versus the density fluctuation amplitude and the reflected signal seems to be more homogeneous (less bursty). The spreading of the beam by forward scattering in the case of Tore Supra seems to be small but the path in the plasma in ITER will be long enough to require that the contribution of forward scattering have to be taken into account to interpret correctly the results.

The density fluctuation profile becomes difficult to reconstruct when the experimental phase has a lot of phase jumps, which is not completely explained at this moment. Such phase jumps have been also seen in simulations. Different configurations are able to generate phase jumps, non-linear Bragg scattering ${ }^{11}$, transient secondary cut-offs ${ }^{12}$, destructive interference ${ }^{13}$ and wave trapping. This last effect has been recently identified. To illustrate the effect of wave trapping, an academic configuration is used. First, using a coherent density perturbation with high amplitude satisfying the resonant Bragg backscattering rule a resonant cavity is generated in a linear density profile. Then, a moving density perturbation is put in the cavity and the phase variation is computed as a function of the position of the density perturbation. To have significant variations of the phase the secondary density perturbation satisfies the Bragg backscattering at a given position in the resonant cavity. The density perturbations have a Gaussian shape with a spatial sinusoidal modulation (see fig 1_a).
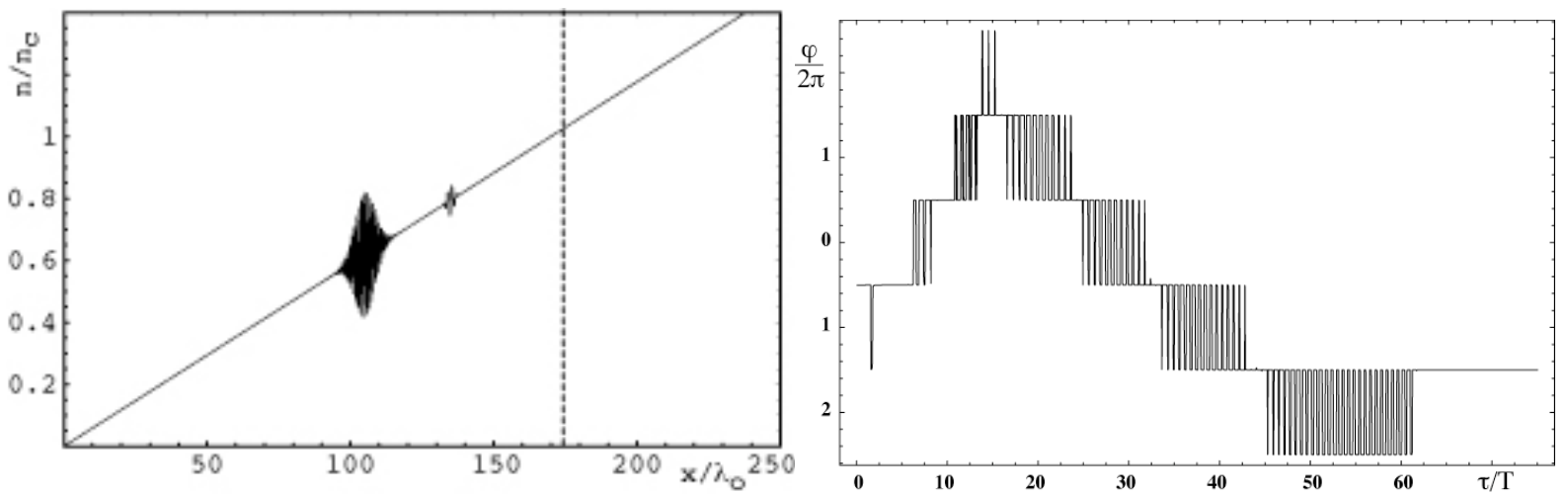

figure 1_a: density profile including both Gaussian density perturbations a) constituting the resonant cavity $\beta$ ) moving density perturbation able to generate phase jumps. Figure $1 \_b$ : Phase variations in the case of non-linear behavior with phase jumps.

To obtain phase jumps, the quality factor of the cavity needs to be high enough to saturate the phase variation (see fig. 1_b). Each phase jump can be fully described by changing the space step size. The global shape corresponds to the non-linear phase behavior of the density perturbation without cavity effects. The resonant cavity effect can be associated to the discrete step of the phase variation. In plasma such resonant conditions can be reached from time to time and give phase jumps. In solid state physics, these conditions can be defined more properly than in plasma physics and resonant conditions can be used to amplify the sensitivity as it is shown (see fig 1_b).

The dynamics of the turbulence becomes an interesting subject due to improvements in hardware, which offeres now the possibilities to sweep the frequency bandwidth in less than $4 \mu$ s plus a dead time of $1 \mu \mathrm{s}$ and allowing a sampling rate of $200 \mathrm{kHz}$. Then fast single 
events associated to turbulence transport can be studied. With a frequency sweep time smaller than the correlation time of the turbulence, the density fluctuation can be considered frozen and in this case the wavenumber spectrum up to the Bragg limit can be reached.

Acknowledgements : Financial support of RFBR grants 06-02-17212, 07-02-92162-CNRS, NWO-RFBR grant 047.016.015 and INTAS grant 05-8046 is acknowledged.

\section{Bibliography :}

1 R. Sabot, C. Bottereau, J.-M. Chareau, F. Clairet, F. Gabillet, P. Hennequin, S. Heuraux, C. Honore, G. Leclert, "Advances of reflectometry on Tore-Supra from edge density profile to core density fluctuations", International Journal of Infrared and Millimeter Waves 25 229-243 (2004).

2 E. Z. Gusakov, G. Leclert, I. Boucher, S. Heuraux, S. Hacquin, M. Colin, V V Bulanin, A. V. Petrov, B. O. Yakovlev, F. Clairet and X. L. Zou "Small angle scattering and spatial resolution of fluctuation reflectometry: comparison of the two-dimensional analytical theory with numerical calculations" Plasma Phys Control. Fusion 44, 1565 (2002).

3 G. Leclert, S. Heuraux, E. Z. Gusakov, A.Yu. Popov, I. Boucher, L. Vermare "Full-wave test of the radial correlation reflectometry analytical theory in linear and nonlinear regime" Plasma Physics Control. Fusion 48, 1389 (2006).

4 M. Schubert, S. Heuraux, T. Gerbaud, F. Clairet, R. Sabot, M. Hirsch "On the absolute value of the density fluctuation determined by fluctuation reflectometry: role of the wavenumber spectrum of the density fluctuation" $34^{\text {th }}$ EPS Conf. controlled fusion and plasma physics Warsaw (Poland) 3-7 July 2007, website //epsppd.epfl.ch / P5090.

5 E.Z. Gusakov, S. Heuraux, N.V. Kosolapova, A. Yu. Popov "Turbulence characterisation in tokamak using radial correlation reflectometry data" NAMES 2007 ARCUS $3^{\text {rd }}$ Russia-France, Metz, 7-9 November, this conference.

6 S. Heuraux, S. Hacquin, F. da Silva, F. Clairet, R. Sabot G. Leclert "Radial wave number spectrum of the density fluctuations deduced from phase reflectometry signals" Rev. Sci. Instrum. 74, 1501-1506(2003).

7 L. Vermare, S. Heuraux, F. Clairet, G.Leclert, F. da Silva "Density fluctuations measurements using X-mode fast sweep reflectometry on Tore Supra" Nuclear Fusion 46, S743 (2006).

8 T. Gerbaud, F. Clairet, A. Sirinelli, R.. Sabot, S. Heuraux, L. Vermare, G. Leclert "Comparison of density fluctuations measurement between O-mode and X-mode reflectometry on Tore Supra" Rev. Sci. Instrum. 77, 10E928 (2006).

9 Ph. Moreau, F. Clairet, J. M. Chareau, M. Paume, C. Laviron "Ultrafast frequency sweep heterodyne reflectometer on the Tore Supra tokamak Rev. Sci.Instrum.71, 74(2000).

10 B. B. Afeyan, A. E . Chou, B. I .Cohen. "The scattering phase shift due to Bragg resonance in one-dimensional fluctuation reflectometry" Plasma Phys. Control. Fusion, 37, 315 (1995).

11 I. Boucher, C. Fanack, S. Heuraux, G. Leclert, F. Clairet, and X.L. Zou "One-dimensional analytical model of the phase shift due to Bragg backscattering of an ordinary wave by large amplitude density fluctuations" Plasma Phys. Control. Fusion 40, 1489 (1998).

12 L. Vermare, F. Clairet, S. Heuraux, G. Leclert "Rational surfaces localization and MHD activity measurements using fast sweep reflectometry" Plasma Phys. Cont. Fusion 47, 1895 (2005).

13 F. da Silva, S. Heuraux, S. Hacquin, M.E. Manso "Simulation of amplitude variations induced by magnetic islands with turbulence on reflectometry signals" Rev. Sci. Instrum. 74, 1497-1501 (2003).

14 E. Z Gusakov,A Yu Popov "Two-dimensional non-linear theory of radial correlation reflectometry" Plasma Phys. Control. Fusion 461393 (2004).

15 E. Z. Gusakov, A.V. Surkov, A. Yu Popov "Multiple scattering effect in Doppler reflectometry" Plasma Phys. Control. Fusion 47959 (2005). 